Supporting Information Available

\title{
Reaction of Hydroxyl radicals with Azacytosines: A Pulse Radiolysis and Theoretical Study \\ G. Pramod, ${ }^{1}$ K.P. Prasanthkumar, ${ }^{1}$ Hari Mohan, ${ }^{2}$ V.M.Manoj, ${ }^{1}$ P.Manoj, ${ }^{1}$ C.H. Suresh, ${ }^{3 *}$ and C.T. Aravindakumar ${ }^{1 *}$
}

1. School of Chemical Sciences, Mahatma Gandhi University, Kottayam 686560, India

2. Radiation Chemistry and Chemical Dynamics Division, Bhabha Atomic Research Centre, Mumbai 400085, India

3. Computational Modeling and Simulation Section, Regional Research Laboratory (CSIR), Trivandrum 695 019, India

Contents:

1. Optimized geometries and the corresponding thermodynamic quantities.

2. A figure showing the B3LYP/6-31+G $(\mathrm{d}, \mathrm{p})$ level optimized geometries of $\mathrm{H}$ abstraction product radicals. Bond lengths are in $\AA$ units 
1. Optimized geometries and the corresponding thermodynamic quantities.

All the geometries were optimized using the B3LYP method with $6-31+\mathrm{G}(\mathrm{d}, \mathrm{p})$ basis set. Also the 6D function has been used throughout the calculations. Standard orientations are given in Cartesian coordinate format in Angstrom unit (Atomic number, $\mathrm{X}, \mathrm{Y}$, and Z coordinates).

\section{5-AZACYTOSINE}

$\begin{array}{llll}7 & 1.098652 & 1.136827 & -0.000139\end{array}$

$\begin{array}{llll}6 & -0.092418 & 1.658533 & 0.000173\end{array}$

$\begin{array}{llll}7 & -1.223529 & 0.925527 & 0.000046\end{array}$

$6 \quad-1.193984-0.511226-0.000408$

$7 \quad 0.057601 \quad-1.058969-0.000222$

$6 \quad 1.103951 \quad-0.251630-0.000440$

$1 \quad-0.201637 \quad 2.741262 \quad 0.000205$

$1 \quad-2.139381 \quad 1.357232 \quad 0.000258$

$\begin{array}{llll}8 & -2.254407 & -1.112924 & 0.000327\end{array}$

$\begin{array}{llll}7 & 2.337484 & -0.798538 & 0.000277\end{array}$

$\begin{array}{llll}1 & 2.431361 & -1.801878 & 0.000422\end{array}$

$\begin{array}{llll}1 & 3.148163 & -0.201220 & 0.000817\end{array}$ 
SCF Energy $=-411.01518$ a.u

Zero-point correction $=0.086983($ Hartree/Particle $)$

Thermal correction to Energy $=0.093555$

Thermal correction to Enthalpy=0.094499

Thermal correction to Gibbs free energy $=0.056224$

Sum of electronic and zero-point Energies $=-410.928203$

Sum of electronic and thermal Energies $=-410.921631$

Sum of electronic and thermal Enthalpies $=-410.920687$

Sum of electronic and thermal Free Energies $=-410.958962$

\section{CYTOSINE}

$\begin{array}{llll}7 & -0.082130 & -1.051213 & 0.001455\end{array}$

$\begin{array}{llll}6 & 1.185443 & -0.529002 & 0.000090\end{array}$

$\begin{array}{llll}7 & 1.283745 & 0.894642 & 0.001311\end{array}$

$\begin{array}{llll}6 & 0.203978 & 1.715265 & 0.000977\end{array}$

$6 \quad-1.050358 \quad 1.187994 \quad-0.000851$

$\begin{array}{lllll}6 & -1.133999 & -0.251357 & -0.001587\end{array}$ 


$\begin{array}{lllll}7 & -2.359227 & -0.843714 & -0.025152 \\ 8 & 2.222141 & -1.179607 & 0.000674 \\ & & & & \\ 1 & 2.226813 & 1.260221 & 0.002529 \\ & & & \\ 1 & 0.397798 & 2.782769 & 0.001288 \\ 1 & -1.929290 & 1.819752 & -0.007603 \\ & & & & \\ 1 & -3.203797 & -0.312278 & 0.106238 \\ 1 & -2.395752 & -1.849008 & 0.057085\end{array}$

SCF Energy = -394.96316 a.u

Zero-point correction $=0.098172($ Hartree/Particle $)$

Thermal correction to Energy= 0.105030

Thermal correction to Enthalpy=0.105974

Thermal correction to Gibbs free energy $=0.067196$

Sum of electronic and zero-point Energies $=-394.864991$

Sum of electronic and thermal Energies $=-394.858133$

Sum of electronic and thermal Enthalpies= -394.857189

Sum of electronic and thermal Free Energies=-394.895967 


\section{ADDUCT SYSTEMS}

\begin{tabular}{|c|c|c|c|}
\hline \multicolumn{4}{|c|}{ N3-OH_5-Ac } \\
\hline 7 & -0.993035 & 1.505461 & -0.029893 \\
\hline 6 & 0.251041 & 2.007472 & 0.084792 \\
\hline 7 & 1.364089 & 1.139287 & -0.015148 \\
\hline 6 & 1.234035 & -0.212194 & 0.012387 \\
\hline 7 & -0.072560 & -0.640545 & 0.331038 \\
\hline 6 & -1.156870 & 0.212422 & 0.009553 \\
\hline 1 & 0.440638 & 3.066517 & 0.001186 \\
\hline 1 & 2.297590 & 1.495188 & -0.169575 \\
\hline 8 & 2.123158 & -1.041254 & -0.174333 \\
\hline 7 & -2.363049 & -0.393097 & -0.195164 \\
\hline 1 & -3.168194 & 0.214018 & -0.159786 \\
\hline 1 & -2.477888 & -1.351522 & 0.098962 \\
\hline 8 & -0.279442 & -2.011860 & 0.055694 \\
\hline 1 & 0.640771 & -2.323231 & -0.097905 \\
\hline
\end{tabular}


SCF Energy $=-486.74204$ a.u

Zero-point correction=0.099339 (Hartree/Particle)

Thermal correction to Energy= 0.107873

Thermal correction to Enthalpy $=0.108817$

Thermal correction to Gibbs free energy $=0.065915$

Sum of electronic and zero-point Energies $=-486.642703$

Sum of electronic and thermal Energies $=-486.634169$

Sum of electronic and thermal Enthalpies $=-486.633225$

Sum of electronic and thermal Free Energies $=-486.676126$

C4-OH_5-Ac

7

$\begin{array}{lll}0.871115 & 1.259447 & 0.003254\end{array}$

6

$\begin{array}{lll}-0.323085 & 1.711812 & 0.035715\end{array}$

7

$\begin{array}{lll}-1.457600 & 0.932786 & 0.054658\end{array}$

6

$\begin{array}{lll}-1.408464 & -0.466787 & -0.029498\end{array}$ 


$\begin{array}{lllll}7 & -0.157079 & -1.050844 & -0.116598 \\ 6 & 1.020538 & -0.189601 & -0.019967 \\ 1 & -0.494845 & 2.786912 & 0.049980 \\ 1 & -2.376776 & 1.352807 & 0.088712 \\ 8 & -2.441906 & -1.125220 & -0.040794 \\ 7 & 1.591335 & -0.586784 & 1.230739 \\ 1 & 1.936415 & -1.539739 & 1.215971 \\ 1 & 2.252428 & 0.082222 & 1.609381 \\ 8 & 1.788673 & -0.522808 & -1.164103 \\ 1 & 2.240313 & 0.287234 & -1.446736\end{array}$

SCF Energy $=-486.75893$ a.u

Zero-point correction= $0.100081($ Hartree/Particle $)$

Thermal correction to Energy= 0.108243

Thermal correction to Enthalpy $=0.109187$

Thermal correction to Gibbs free energy $=0.066081$

Sum of electronic and zero-point Energies $=-486.658858$ 
Sum of electronic and thermal Energies $=-486.650696$

Sum of electronic and thermal Enthalpies $=-486.649752$

Sum of electronic and thermal Free Energies $=-486.692858$

N5-OH_5-Ac

7

$-0.995480 \quad-0.546770 \quad-0.244581$

6

$-0.077534 \quad-1.574166 \quad 0.029553$

7

$1.240133 \quad-1.136394 \quad 0.032142$

6

$\begin{array}{lll}1.643378 & 0.212390 & 0.016127\end{array}$

7

$\begin{array}{lll}0.634265 & 1.160941 & -0.024873\end{array}$

6

$\begin{array}{lll}-0.613019 & 0.774492 & -0.059240\end{array}$

$1 \quad-0.300421 \quad-2.534998 \quad-0.428043$

$1 \quad 1.987237 \quad-1.811988 \quad 0.118137$

$\begin{array}{llll}8 & 2.833968 & 0.482499 & 0.044222\end{array}$ 


$\begin{array}{llll}7 & -1.615842 & 1.675577 & 0.058405 \\ 1 & -1.346809 & 2.647415 & 0.030616 \\ 8 & -2.334487 & -0.869311 & 0.054073 \\ 1 & -2.331752 & -1.163446 & 0.987824 \\ 1 & -2.562579 & 1.407731 & -0.161185\end{array}$

SCF Energy $=-486.72771$ a.u

Zero-point correction $=0.100168($ Hartree/Particle $)$

Thermal correction to Energy $=0.108529$

Thermal correction to Enthalpy= 0.109473

Thermal correction to Gibbs free energy $=0.066670$

Sum of electronic and zero-point Energies $=-486.627544$

Sum of electronic and thermal Energies $=-486.619183$

Sum of electronic and thermal Enthalpies $=-486.618238$

Sum of electronic and thermal Free Energies $=-486.661041$ 
C6-OH_5-Ac

\begin{tabular}{|c|c|c|c|}
\hline 7 & -0.296644 & -1.318515 & 0.035067 \\
\hline 6 & 1.031495 & -0.898346 & 0.370392 \\
\hline 7 & 1.242815 & 0.514362 & 0.124128 \\
\hline 6 & 0.226192 & 1.440953 & -0.024049 \\
\hline 7 & -1.070942 & 0.929065 & -0.182927 \\
\hline 6 & -1.268308 & -0.378674 & -0.000180 \\
\hline 1 & 1.177902 & -1.135969 & 1.439678 \\
\hline 1 & 2.153085 & 0.896579 & 0.348837 \\
\hline 8 & 0.442994 & 2.643160 & -0.043256 \\
\hline 7 & -2.550146 & -0.827325 & 0.050006 \\
\hline 1 & -3.286019 & -0.185524 & -0.204684 \\
\hline 1 & -2.714851 & -1.819237 & -0.028215 \\
\hline 8 & 2.005922 & -1.671441 & -0.279331 \\
\hline 1 & 1.866698 & -1.596299 & -1.235843 \\
\hline
\end{tabular}


SCF Energy = -486.77774 a.u

Zero-point correction $=0.099724($ Hartree/Particle $)$

Thermal correction to Energy $=0.108107$

Thermal correction to Enthalpy $=0.109051$

Thermal correction to Gibbs free energy $=0.065440$

Sum of electronic and zero-point Energies $=-486.678018$

Sum of electronic and thermal Energies $=-486.669635$

Sum of electronic and thermal Enthalpies $=-486.668691$

Sum of electronic and thermal Free Energies=-486.712302 


\title{
1, 2-HYDROGEN MIGRATION
}

\author{
C6-OH N5H_5-Ac
}

$7 \quad-0.221302 \quad-1.267440 \quad-0.057613$

$6 \quad 1.097689-0.816786 \quad-0.318497$

$\begin{array}{llll}7 & 1.245960 & 0.537620 & -0.008406\end{array}$

$\begin{array}{llll}6 & 0.172930 & 1.461420 & 0.010325\end{array}$

$\begin{array}{llll}7 & -1.106583 & 0.931893 & 0.057750\end{array}$

$6 \quad-1.255771 \quad-0.362681 \quad 0.006170$

$\begin{array}{lllll}1 & 2.148728 & 0.954287 & -0.200993\end{array}$

$\begin{array}{llll}8 & 0.415321 & 2.656345 & 0.020605\end{array}$

$\begin{array}{llll}7 & -2.520535 & -0.886773 & -0.015498\end{array}$

$1 \quad-3.247531 \quad-0.215646 \quad 0.191837$

$\begin{array}{llll}1 & -2.674732 & -1.823853 & 0.327477\end{array}$

$\begin{array}{llll}8 & 2.095012 & -1.658922 & 0.097461\end{array}$

$1 \quad 2.233664 \quad-1.575915 \quad 1.063685$

$1 \quad-0.414651 \quad-2.217072 \quad-0.348149$ 
SCF Energy = -486.79058 a.u

Zero-point correction $=0.101213($ Hartree/Particle $)$

Thermal correction to Energy $=0.109434$

Thermal correction to Enthalpy $=0.110378$

Thermal correction to Gibbs free energy $=0.067841$

Sum of electronic and zero-point Energies $=-486.689374$

Sum of electronic and thermal Energies $=-486.681153$

Sum of electronic and thermal Enthalpies $=-486.680209$

Sum of electronic and thermal Free Energies=-486.722746

TS1

$\begin{array}{llll}7 & 1.125398 & 0.910528 & -0.058160 \\ 6 & -0.135001 & 1.468107 & -0.008789 \\ 7 & -1.233677 & 0.566656 & 0.047628 \\ 6 & -1.076740 & -0.789219 & 0.217834 \\ 7 & & & \\ 7 & 0.224939 & -1.345989 & 0.034344\end{array}$




$\begin{array}{lllll}6 & 1.247936 & -0.396924 & -0.027658 \\ 7 & 2.492604 & -0.938250 & -0.004696 \\ 8 & -0.364519 & 2.663548 & -0.029809 \\ 8 & -2.142527 & -1.531225 & -0.203463 \\ 1 & -2.154506 & 0.978216 & 0.128767 \\ 1 & -0.291710 & -1.388212 & 1.181842 \\ 1 & & & & \\ & 2.604622 & -1.923992 & -0.182413 \\ 1 & 3.269894 & -0.316370 & -0.169795 \\ 1 & -1.853957 & -2.450636 & -0.314358\end{array}$

SCF Energy $=-486.72318$ a.u

Zero-point correction= $0.095228($ Hartree/Particle $)$

Thermal correction to Energy $=0.103416$

Thermal correction to Enthalpy= 0.104360

Thermal correction to Gibbs free energy $=0.061701$

Sum of electronic and zero-point Energies $=-486.627958$

Sum of electronic and thermal Energies $=-486.619771$ 
Sum of electronic and thermal Enthalpies $=-486.618826$

Sum of electronic and thermal Free Energies $=-486.661485$

\section{$\underline{\text { H ABSTRACTION REACTIONS }}$}

\section{HYDROGEN BONDED COMPLEXES}

S1

$\begin{array}{llll}7 & 1.513199 & 1.229101 & -0.000608\end{array}$

$\begin{array}{llll}6 & 0.266395 & 1.603315 & -0.000936\end{array}$

$\begin{array}{llll}7 & -0.770332 & 0.742890 & -0.000593\end{array}$

$6 \quad-0.556071 \quad-0.664770 \quad-0.001033$

$7 \quad 0.738110-1.071230 \quad-0.001121$

$\begin{array}{llll}6 & 1.686525 & -0.144108 & 0.000049\end{array}$ 


$\begin{array}{lllll}8 & -1.538370 & -1.412309 & -0.001382 \\ 7 & 2.971423 & -0.547318 & 0.002786 \\ 1 & 0.026291 & 2.664196 & -0.001447 \\ 1 & -1.743757 & 1.056292 & -0.000633 \\ 1 & 3.178998 & -1.533729 & 0.000822 \\ 1 & 3.709729 & 0.137913 & 0.000450 \\ 8 & -3.665463 & 0.283662 & 0.002503 \\ 1 & -3.088490 & -0.536225 & 0.000106\end{array}$

SCF Energy $=-486.76694$ a.u

Zero-point correction=0.098657 (Hartree/Particle)

Thermal correction to Energy $=0.107485$

Thermal correction to Enthalpy $=0.108429$

Thermal correction to Gibbs free energy $=0.063477$

Sum of electronic and zero-point Energies $=-486.668290$

Sum of electronic and thermal Energies $=-486.659462$

Sum of electronic and thermal Enthalpies $=-486.658518$ 
Sum of electronic and thermal Free Energies $=-486.703470$

S2 (for H10 abstraction)

\begin{tabular}{|c|c|c|c|}
\hline 5 & 1.766924 & -0.189727 & 0.009591 \\
\hline 6 & -0.433242 & -1.224761 & -0.026601 \\
\hline 6 & -0.203176 & 1.029889 & -0.001343 \\
\hline 7 & 1.116567 & 1.013594 & 0.006892 \\
\hline & -1.042050 & -0.074587 & 0.006399 \\
\hline & 0.905613 & -1.341806 & -0.027450 \\
\hline 7 & -0.840398 & 2.219939 & -0.002721 \\
\hline & 2.971688 & -0.365962 & 0.032996 \\
\hline 1 & -1.032794 & -2.130708 & -0.045523 \\
\hline 1 & 1.359937 & -2.246717 & -0.050301 \\
\hline 1 & -0.290706 & 3.060181 & -0.096080 \\
\hline 1 & -1.836811 & 2.246849 & -0.150940 \\
\hline & -3.292525 & -0.990473 & -0.058268 \\
\hline
\end{tabular}


SCF Energy $=-486.76120 \mathrm{a} . \mathrm{u}$

Zero-point correction $=0.097698$ (Hartree/Particle)

Thermal correction to Energy $=0.107342$

Thermal correction to Enthalpy $=0.108287$

Thermal correction to Gibbs free energy $=0.061041$

Sum of electronic and zero-point Energies $=-486.663502$

Sum of electronic and thermal Energies $=-486.653858$

Sum of electronic and thermal Enthalpies $=-486.652914$

Sum of electronic and thermal Free Energies $=-486.700160$

S3 (for H11 abstraction)

6

$\begin{array}{lll}0.763267 & 1.066905 & -0.051314\end{array}$

6

$$
\begin{array}{lll}
1.751836 & -1.148738 & 0.100394
\end{array}
$$




\begin{tabular}{|c|c|c|c|}
\hline 6 & -0.487089 & -0.906734 & -0.070196 \\
\hline 7 & -0.434060 & 0.419260 & -0.129465 \\
\hline 7 & 0.605363 & -1.756449 & 0.028266 \\
\hline 7 & 1.892037 & 0.193822 & 0.075983 \\
\hline 7 & -1.685402 & -1.497769 & -0.118315 \\
\hline 8 & 0.943583 & 2.271978 & -0.087767 \\
\hline 1 & 2.662490 & -1.737815 & 0.186787 \\
\hline 1 & 2.800786 & 0.636782 & 0.136250 \\
\hline 1 & -2.500479 & -0.891188 & -0.116592 \\
\hline 1 & -1.749407 & -2.499861 & -0.040564 \\
\hline 8 & -2.657380 & 1.107937 & 0.240751 \\
\hline 1 & -2.316655 & 1.872118 & -0.258341 \\
\hline
\end{tabular}

(1) $-2.316655-1.872118-0.258341$

SCF Energy $=-486.76977$ a.u

Zero-point correction= $0.098625($ Hartree/Particle $)$

Thermal correction to Energy= 0.107730

Thermal correction to Enthalpy= 0.108674 
Thermal correction to Gibbs free energy $=0.063631$

Sum of electronic and zero-point Energies=-486.671146

Sum of electronic and thermal Energies $=-486.662041$

Sum of electronic and thermal Enthalpies $=-486.661097$

Sum of electronic and thermal Free Energies $=-486.706140$

S4 (for H12 abstraction)

$\begin{array}{llll}6 & 1.845295 & -0.195683 & -0.000146\end{array}$

$\begin{array}{llll}6 & 0.122908 & 1.524576 & 0.000280\end{array}$

$\begin{array}{llll}6 & -0.419201 & -0.681797 & 0.000205\end{array}$

$7 \quad 0.831152-1.108147 \quad-0.000122$

$\begin{array}{llll}7 & -0.842173 & 0.648954 & 0.000415\end{array}$

$7 \quad 1.420866 \quad 1.183563 \quad 0.000031$

$\begin{array}{llll}7 & -1.415980 & -1.584591 & 0.000505\end{array}$ 


$$
\begin{array}{llll}
8 & 3.040510 & -0.426829 & -0.000358 \\
1 & -0.118968 & 2.585228 & 0.000391 \\
1 & 2.155267 & 1.881039 & -0.000064 \\
1 & -1.175707 & -2.563860 & -0.000248 \\
1 & -2.383844 & -1.283891 & -0.000227 \\
8 & -3.666876 & 0.514331 & -0.000626 \\
1 & -2.716884 & 0.820448 & 0.000187
\end{array}
$$

SCF Energy $=-486.76732$ a.u

Zero-point correction $=0.098714($ Hartree/Particle $)$

Thermal correction to Energy $=0.107525$

Thermal correction to Enthalpy $=0.108469$

Thermal correction to Gibbs free energy $=0.063702$

Sum of electronic and zero-point Energies $=-486.668608$

Sum of electronic and thermal Energies $=-486.659798$

Sum of electronic and thermal Enthalpies $=-486.658853$

Sum of electronic and thermal Free Energies $=-486.703621$ 


\section{TRANSITION STATES FOR H-ABSTRACTION REACTIONS}

QST3 method is used for optimizations.

TS2

\begin{tabular}{|c|c|c|c|}
\hline 6 & 0.582454 & -0.593648 & 0.000604 \\
\hline & -0.205343 & 1.593315 & 0.012815 \\
\hline & -1.636613 & -0.146605 & -0.000594 \\
\hline & -0.669922 & -1.073184 & -0.006281 \\
\hline & -1.464888 & 1.211440 & 0.009063 \\
\hline & 0.840621 & 0.761214 & 0.011545 \\
\hline & -2.912647 & -0.578236 & -0.004585 \\
\hline & 1.600257 & -1.368032 & 0.008301 \\
\hline & 0.011118 & 2.659213 & 0.017700 \\
\hline & 2.083240 & 0.791100 & 0.008234 \\
\hline
\end{tabular}




$\begin{array}{lllll}1 & -3.663934 & 0.092516 & -0.020573 \\ 1 & -3.100557 & -1.568027 & -0.029175 \\ 8 & 3.174567 & 0.222944 & -0.113095 \\ 1 & & 3.476406 & -0.181117 & 0.717031\end{array}$

SCF Energy $=-486.76203$ a.u

Zero-point correction=0.094962 (Hartree/Particle)

Thermal correction to Energy= 0.103061

Thermal correction to Enthalpy $=0.104005$

Thermal correction to Gibbs free energy $=0.061590$

Sum of electronic and zero-point Energies=- 486.667073

Sum of electronic and thermal Energies $=-486.658975$

Sum of electronic and thermal Enthalpies $=-486.658030$

Sum of electronic and thermal Free Energies $=-486.700445$ 
TS3

\begin{tabular}{|c|c|c|c|}
\hline 7 & 0.290352 & -1.251661 & -0.043891 \\
\hline 6 & 1.061962 & -0.229829 & -0.058365 \\
\hline 7 & 0.640664 & 1.038798 & -0.039903 \\
\hline 6 & -0.776772 & 1.345337 & 0.007480 \\
\hline 7 & -1.595967 & 0.251562 & 0.026756 \\
\hline 6 & -1.071009 & -0.956020 & 0.000404 \\
\hline 1 & 2.283708 & -0.350319 & -0.109019 \\
\hline 1 & 1.285403 & 1.820840 & -0.041742 \\
\hline 8 & -1.105368 & 2.514951 & 0.025790 \\
\hline 7 & -1.883195 & -2.028911 & 0.015641 \\
\hline 1 & -1.488056 & -2.955137 & -0.006099 \\
\hline 1 & -2.881707 & -1.893025 & 0.044652 \\
\hline 1 & 3.727415 & -1.220245 & 0.347910 \\
\hline & 3.558513 & -0.318397 & 0.018831 \\
\hline
\end{tabular}


SCF Energy = -486.75535 a.u

Zero-point correction $=0.092409($ Hartree/Particle $)$

Thermal correction to Energy $=0.101505$

Thermal correction to Enthalpy $=0.102449$

Thermal correction to Gibbs free energy $=0.056659$

Sum of electronic and zero-point Energies $=\quad-486.662948$

Sum of electronic and thermal Energies $=\quad-486.653852$

Sum of electronic and thermal Enthalpies $=\quad-486.652908$

Sum of electronic and thermal Free Energies $=\quad-486.698697$

TS4

$\begin{array}{llll}6 & -0.877784 & 1.006090 & 0.048652\end{array}$

$6 \quad-1.594856-1.320153-0.078882$

$\begin{array}{llll}6 & 0.627071 & -0.864120 & 0.101434\end{array}$

$\begin{array}{llll}7 & 0.347364 & 0.461471 & 0.291822\end{array}$ 


$\begin{array}{lllll}7 & -0.398348 & -1.806612 & 0.027087 \\ 7 & -1.888885 & 0.004342 & -0.103235 \\ 7 & 1.887012 & -1.178345 & 0.014507 \\ 8 & -1.174237 & 2.185032 & 0.007652 \\ 1 & -2.438005 & -2.002699 & -0.165342 \\ 1 & -2.836918 & 0.339509 & -0.222865 \\ 1 & 2.450654 & 0.014272 & -0.106847 \\ 1 & 2.089591 & -2.163328 & -0.106644 \\ 8 & 2.390907 & 1.175146 & -0.261369 \\ 1 & 2.444732 & 1.633926 & 0.592950\end{array}$

SCF Energy $=-486.74952$ a.u

Zero-point correction=0.095066 (Hartree/Particle $)$

Thermal correction to Energy= 0.102961

Thermal correction to Enthalpy $=0.103905$

Thermal correction to Gibbs free energy $=0.061616$

Sum of electronic and zero-point Energies $=-486.654462$ 
Sum of electronic and thermal Energies $=-486.646567$

Sum of electronic and thermal Enthalpies $=-486.645623$

Sum of electronic and thermal Free Energies $=-486.687913$

TS5

$\begin{array}{llll}6 & -1.771995 & -0.438884 & 0.099462\end{array}$

$\begin{array}{llll}6 & -0.530111 & 1.641194 & -0.107755\end{array}$

$\begin{array}{llll}6 & 0.530574 & -0.346373 & -0.201639\end{array}$

$7 \quad-0.554988 \quad-1.076770 \quad 0.002631$

$\begin{array}{llll}7 & 0.613861 & 1.037761 & -0.236158\end{array}$

$\begin{array}{llll}7 & -1.703938 & 0.987395 & 0.030785\end{array}$

$7 \quad 1.694416 \quad-1.001169 \quad-0.458569$

$\begin{array}{llll}8 & -2.851330 & -0.978696 & 0.251214\end{array}$

$1 \quad-0.567943 \quad 2.728415 \quad-0.111799$

$1 \quad-2.580882 \quad 1.486041 \quad 0.125584$

$1 \quad 1.655643 \quad-1.981932 \quad-0.185954$ 

8 $\begin{array}{lll}3.582352 & 0.046047 & 0.454367\end{array}$
$1 \quad 2.729874 \quad-0.444505 \quad-0.284548$
$\begin{array}{llll}1 & 3.198877 & 0.907023 & 0.700845\end{array}$

SCF Energy = -486.75349 a.u

Zero-point correction $=0.093401($ Hartree/Particle $)$

Thermal correction to Energy= 0.101754

Thermal correction to Enthalpy $=0.102698$

Thermal correction to Gibbs free energy $=0.058774$

Sum of electronic and zero-point Energies $=-486.660096$

Sum of electronic and thermal Energies $=-486.651743$

Sum of electronic and thermal Enthalpies $=-486.650799$

Sum of electronic and thermal Free Energies $=-486.694723$ 


\section{HYDROGEN ABSTRACED PRODUCTS}

P1 (H9 abstracted radical)

\begin{tabular}{|c|c|c|c|}
\hline 6 & 1.091656 & -0.229874 & 0.000065 \\
\hline 6 & -1.186177 & -0.407396 & -0.000133 \\
\hline 6 & -0.196435 & 1.651896 & 0.000464 \\
\hline 7 & 0.027557 & -1.034312 & 0.001366 \\
\hline 7 & -1.336774 & 0.969866 & -0.001581 \\
\hline 7 & 2.322970 & -0.781739 & -0.002014 \\
\hline 7 & 1.039165 & 1.137154 & 0.000947 \\
\hline 8 & -2.239016 & -1.111046 & 0.000132 \\
\hline 1 & -0.266009 & 2.737463 & 0.001014 \\
\hline 1 & 2.416506 & -1.785197 & 0.002201 \\
\hline & 3.136941 & -0.188436 & 0.002328 \\
\hline
\end{tabular}

SCF Energy $=-410.32835$ a.u

Zero-point correction $=0.071579($ Hartree $/$ Particle $)$ 
Thermal correction to Energy= 0.078389

Thermal correction to Enthalpy= 0.079334

Thermal correction to Gibbs free energy $=0.038852$

Sum of electronic and zero-point Energies $=-410.256777$

Sum of electronic and thermal Energies $=-410.249966$

Sum of electronic and thermal Enthalpies $=-410.249022$

Sum of electronic and thermal Free Energies $=-410.289503$

P2 (H10 abstracted radical)
$7 \quad-1.065336 \quad 1.182570 \quad-0.000080$
$\begin{array}{llll}6 & 0.099558 & 1.683175 & 0.000213\end{array}$
$7 \quad 1.234941 \quad 0.992136 \quad-0.000058$
$6 \quad 1.195139 \quad-0.472716 \quad-0.000242$
$\begin{array}{llll}7 & -0.061227 & -1.004812 & -0.000195\end{array}$
$6 \quad-1.112725 \quad-0.211915 \quad-0.000554$ 


$\begin{array}{llll}1 & 2.152134 & 1.420953 & 0.000624 \\ 8 & 2.255307 & -1.064667 & 0.000268 \\ 7 & -2.348148 & -0.743213 & 0.000073 \\ 1 & -3.152136 & -0.136225 & 0.001460 \\ 1 & -2.455900 & -1.745428 & 0.001077\end{array}$

SCF Energy $=-410.33764$ a.u

Zero-point correction $=0.073804($ Hartree/Particle $)$

Thermal correction to Energy $=0.080359$

Thermal correction to Enthalpy $=0.081303$

Thermal correction to Gibbs Free Energy= 0.042503

Sum of electronic and zero-point Energies=-410.263836

Sum of electronic and thermal Energies $=-410.257281$

Sum of electronic and thermal Enthalpies $=-410.256337$

Sum of electronic and thermal Free Energies $=-410.295137$ 
P3 (H11 abstracted radical)

\begin{tabular}{|c|c|c|c|}
\hline 6 & -1.188926 & -0.454428 & 0.000021 \\
\hline 6 & 0.043724 & 1.639902 & -0.000082 \\
\hline 6 & 1.152665 & -0.345885 & 0.000008 \\
\hline 7 & 0.044073 & -1.092586 & -0.000037 \\
\hline 7 & 1.198808 & 1.053003 & 0.000264 \\
\hline 7 & -1.133403 & 0.964863 & -0.000296 \\
\hline 7 & 2.322952 & -1.025974 & -0.000043 \\
\hline 8 & -2.263694 & -1.023356 & 0.000122 \\
\hline 1 & -0.014545 & 2.726259 & 0.000355 \\
\hline 1 & -2.021941 & 1.452331 & 0.000165 \\
\hline 1 & 3.074252 & -0.324430 & -0.000387 \\
\hline
\end{tabular}

SCF Energy = -410.32639 a.u

Zero-point correction $=0.072628($ Hartree/Particle $)$

Thermal correction to Energy $=0.078837$ 
Thermal correction to Enthalpy $=0.079781$

Thermal correction to Gibbs free energy $=0.041324$

Sum of electronic and zero-point Energies $=-410.253767$

Sum of electronic and thermal Energies $=-410.247559$

Sum of electronic and thermal Enthalpies $=-410.246614$

Sum of electronic and thermal Free Energies $=-410.285071$

P4 (H12 abstracted radical)

$\begin{array}{llll}6 & 1.164272 & -0.480193 & 0.000096 \\ 6 & 0.007488 & 1.657000 & -0.000103 \\ 6 & -1.177523 & -0.268419 & 0.000075 \\ 7 & -0.097885 & -1.061290 & 0.000465 \\ 7 & & & \\ 7 & -1.171880 & 1.120774 & -0.000264 \\ 7 & & & \\ 7 & 1.160406 & 0.937606 & 0.000266 \\ 7 & -2.405845 & -0.847868 & -0.000167\end{array}$



8
$2.213266 \quad-1.095568 \quad-0.000389$
$\begin{array}{llll}1 & 0.110055 & 2.739995 & 0.000338\end{array}$
$1 \quad 2.066367 \quad 1.392281 \quad 0.000110$
$\begin{array}{llll}1 & -2.241539 & -1.862618 & 0.000152\end{array}$

SCF Energy $=-410.32777$ a.u

Zero-point correction $=0.072648($ Hartree/Particle $)$

Thermal correction to Energy $=0.078928$

Thermal correction to Enthalpy $=0.079872$

Thermal correction to Gibbs Free Energy= 0.041162

Sum of electronic and zero-point Energies $=-410.255123$

Sum of electronic and thermal Energies $=-410.248842$

Sum of electronic and thermal Enthalpies $=-410.247898$

Sum of electronic and thermal Free Energies $=-410.286609$ 
2. A figure showing the B3LYP/6-31+G (d, p) level optimized geometries of $H$ abstraction product radicals. Bond lengths are in $\AA$ units

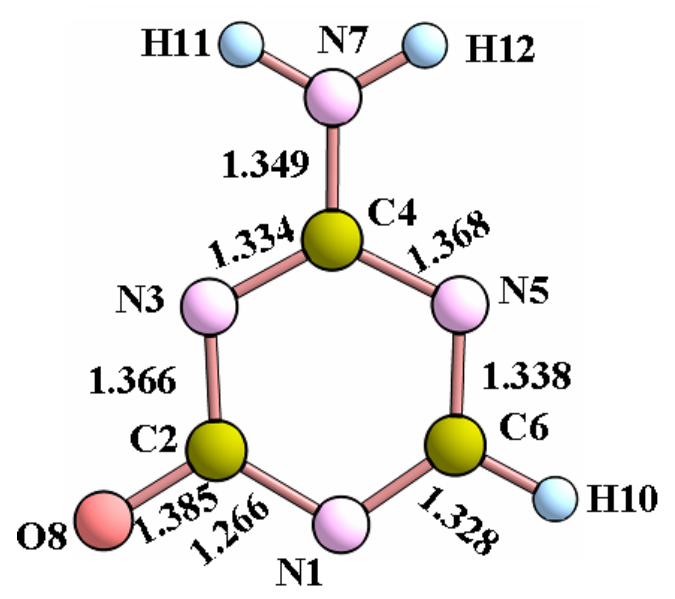

P1

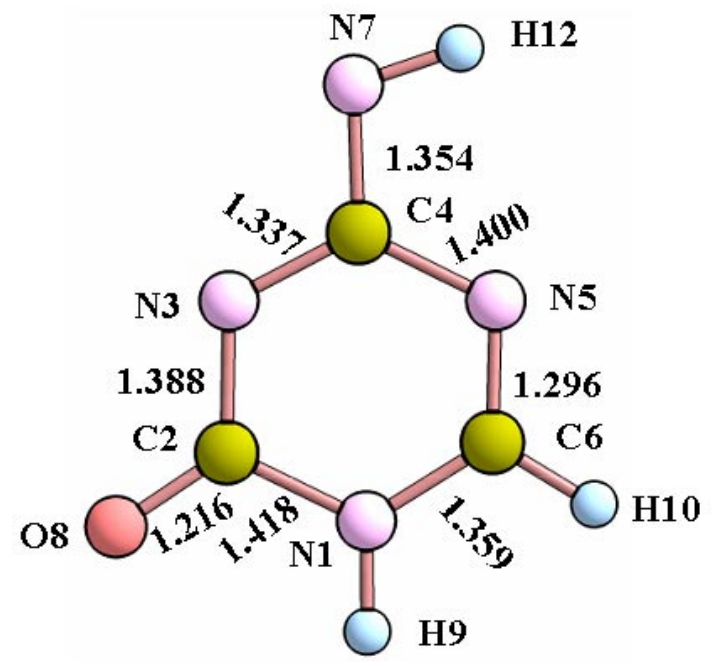

P3

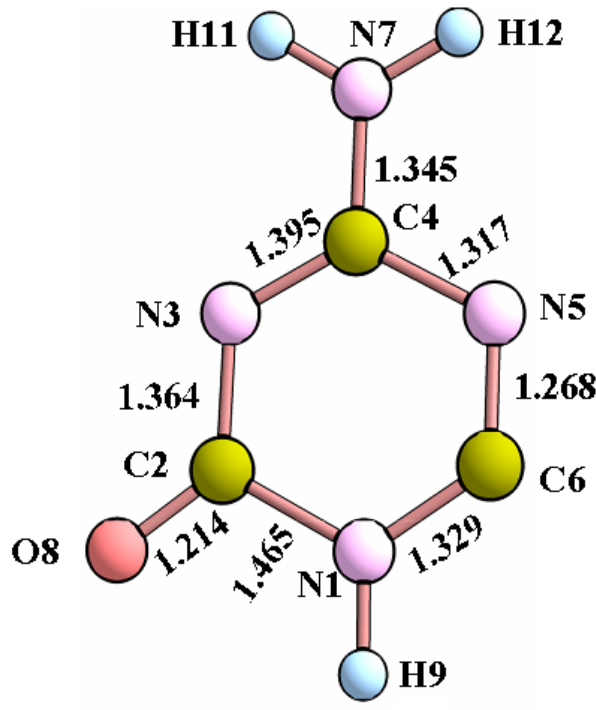

P2

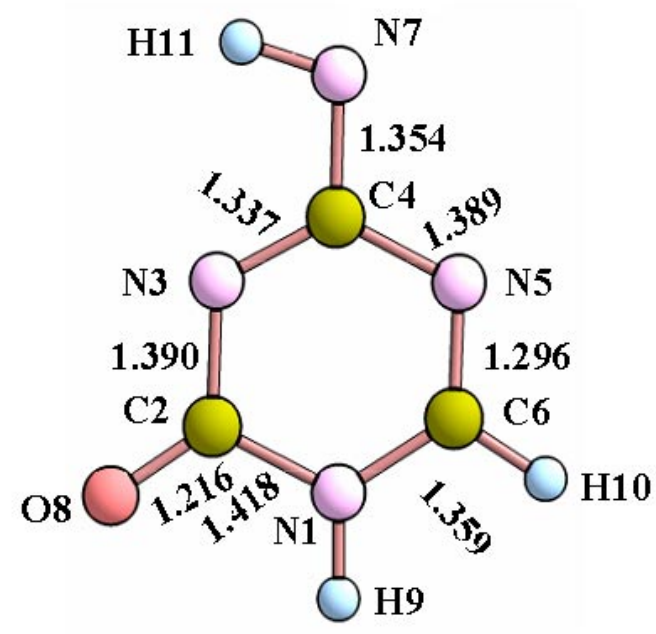

P4 